\title{
DESARROLLO DE APLICACIONES MULTITAREA PARA EL ESTUDIO DE LA MEJORA DEL RENDIMIENTO COGNITIVO EN PACIENTES CON ENFERMEDADES NEURODEGENERATIVAS
}

\author{
Eduardo Garbayo Herce, Montserrat Gil-Martínez \\ egarbayo@unirioja.es,montse.gil@unirioja.es \\ Grupo de Investigación de Ingeniería de Control, Departamento de Ingeniería Eléctrica \\ Universidad de La Rioja, San José de Calasanz 31, 26004 Logroño, España
}

\begin{abstract}
Resumen
La esperanza de vida se ha duplicado en sólo cuatro generaciones, por lo que cómo envejecer conservando buenas facultades físicas y mentales es una de las preocupaciones de la ciencia. El estudio del deterioro cognitivo y cómo paliarlo agrupa diversos campos de trabajo, entre los que figuran los proyectos de entrenamiento cerebral a través de aplicaciones informáticas. Si bien estos estudios están aún en un estado embrionario, ya que se requieren datos y resultados a largo plazo, se vislumbra que este tipo de actividades pueden contribuir positivamente. En este trabajo se presenta cómo se han desarrollado para tal efecto aplicaciones que, aunque basadas en dinámicas clásicas, están siendo diseñadas, programadas, adaptadas y personalizadas para este estudio, buscando unos objetivos concretos. En particular, se han programado unos juegos de -no acción-, con tareas añadidas, independientes y no relacionadas, con el fin de entrenar diferentes facetas y habilidades sometiendo al cerebro a un trabajo extra, que se espera sea positivo y complemente una mejora en el proceso de deterioro cognitivo. Simultáneamente, para el control, seguimiento y examen de dichas competencias, se ha creado una plataforma interactiva en Internet, donde se irán tomando muestras via test online de los progresos.
\end{abstract}

Palabras clave: multitarea, enfermedades degenerativas, entrenamiento mental, superancianos.

\section{INTRODUCCIÓN}

Las enfermedades neurodegenerativas están llamadas a ser la epidemia del siglo XXI, como lo fueron las infecciones hasta que se descubrió la penicilina. Son patologías "nuevas" que hace 50 años no se conocían porque la esperanza de vida era más corta.

La Sociedad Española de Neurología (SEN) estima que existen 800.000 personas en España con enfermedad de Alzheimer y afecta al 5 por ciento de la población mayor de 60 años, al 20 por ciento de los mayores de 80 años y al 30 por ciento de los mayores de 90 años [1].

Igual de importante será tratar el impacto social y, sin caer en la frivolidad, el económico de estas enfermedades neurodegenerativas. Existen estudios [2] de los costes directamente cuantificables, como gasto farmacétuico o utilización de recursos sanitarios, así como de servicios no reembolsados que dedican cuidadores no profesionales dentro del entorno familiar: pérdidas de productividad, etc. En estadísticas publicadas por el IMSERSO el 31 de enero de 2017, se habían presentado 1.625.864 solicitudes y sólo el 71\% (873.706 casos) están recibiendo prestaciones sociales [3].

Un estudio reciente, hecho por expertos del Hospital General de Massachusetts, analiza las causas del envejecimiento cerebral. Como afirma la neurocientífica Emily Rogalski, "una corteza adelgazada sugiere pérdida de neuronas o materia gris" [4]. Por eso, quienes con la edad pierden grosor en esta capa externa del cerebro, sufren un declive en sus funciones cognitivas. Sin embargo, los llamados superancianos logran mantener intactas esas zonas a lo largo de los años. En varios laboratorios se ha podido observar que dichas áreas trabajan más cuando se realizan tareas difíciles que requieren gran esfuerzo físico o mental. Otros estudios en la misma línea de investigación se están llevando a cabo por la Dra. Lisa F. Barrett, en un estudio de los Superancianos [5].

Por otro lado, las tecnologías de la información han experimentado un auge importante dentro del campo de la neurotecnologías [6]. Desde la estandarización de los teléfonos móviles y tabletas, se han popularizado las aplicaciones y juegos basados en puzles y similares, asegurando que un entrenamiento cerebral (Brain Training) es posible [7]. El alcance de estos medios no ha sido en personas de cierta edad, sino fundamentalmente en una juventud $\mathrm{y}$ adolescencia de los denominados "nativos digitales". No obstante, el uso generalizado de aplicaciones de todo tipo va en aumento, como lo demuestran la lista de descargas en las grandes plataformas. Defensores y detractores del Brain Training existen en partes iguales, pero algunos estudios arrojan datos 
esperanzadores [8]. Aunque los detractores no han encontrado ningún efecto negativo o adverso en su uso, afirman que sólo se consigue que el jugador mejore a nivel práctico en esos juegos y habilidades concretas, no produciéndose mejoras significativas a nivel de rendimiento cognitivo general. Sin embargo, en los últimos años las investigaciones médicas sobre los juegos de no-acción (non-action games), enfocados en parte al entretenimiento y en parte al rendimiento cognitivo, han evolucionado hacia la implementación multitarea (multitasking), arrojando resultados relevantes [9].

En este marco, este trabajo presenta unas aplicaciones informáticas que pretenden entrenar el cerebro del usuario sometiéndole a una sobre-estimulación. El fin último es, posteriormente, realizar un estudio de campo con personas mayores o adultos con enfermedades relacionadas con trastornos cognitivos en fase incipiente, con el fin de extraer conclusiones sobre si el entrenamiento mental realizando varias tareas simultáneas es de utilidad para:

- Mejorar el rendimiento cognitivo.

- Prevenir, retrasar o frenar enfermedades relacionadas con la pérdida de memoria.

- Reactivar aptitudes como la memoria o la atención.

- Reparar el deterioro neuronal.

- Incrementar la actividad registrada en las ondas Theta.

- Trabajar la memoria plástica y la capacidad de aprendizaje.

- Mejorar la atención, y las habilidades cognitivas sensoriales espaciales.

Como se detalla en la Sección 2, basándonos en un gestor de contenidos de código abierto, se ha preparado una plataforma online para gestionar todo el trabajo en la nube. En ella se han añadido diferentes cuestionarios que sirven de control para el análisis presente y futuro de los participantes en el estudio. Asimismo, da acceso a los diferenes niveles de aplicaciones informáticas, basadas en dinámicas clásicas de juegos a los que se les han añadido unas tareas extras. Todo el código se presenta como abierto y a nivel de GIT podrá ser compartido para ampliaciones o diferentes "forks", como ya existe en algunas de ellas. La Sección 3 describe cómo se organizarán los estudios de campo.

\section{APLICACIONES MULTITAREA}

\section{$2.1 \quad$ LA PLATAFORMA}

Para el análisis de situación y el seguimiento de cada participante se usan test de control estandarizados y revisados por diferentes asociaciones. Existen algunos modelos que se deben realizar directamente e "in situ" con los participantes. Algunas veces se requiere ver al usuario mover las manos o un lapicero para crear ciertas formas o dibujar un elemento. Por ejemplo, el "Test del Reloj" o la "Figura de Rey Osterrieth". En estos casos es imprescindible un trato directo y la toma de datos en papel, que luego será traslada a la plataforma.

Dado el avance de las últimas tecnologías a nivel de programación multiplataforma de trabajo, algunos apartados que sólo se pensaban desarrollar en su versión offline con los implicados, se han podido desarrollar también a través de Internet. De esta forma, se ha creado una plataforma online para la gestión de los test más complejos o de los que por su planteamiento o resolución es posible.

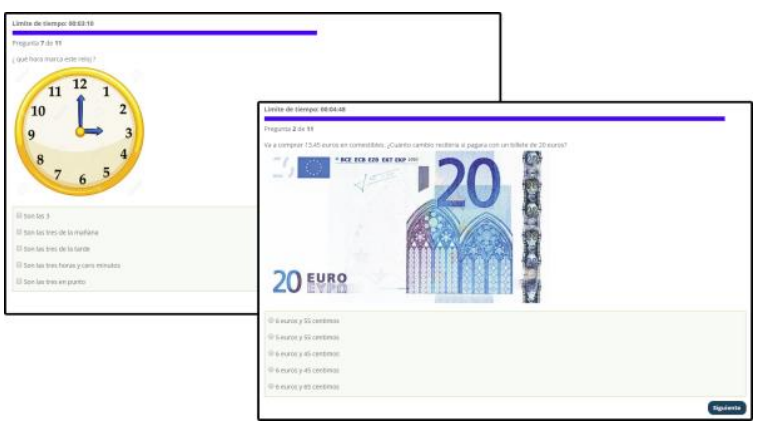

Figura 1. Plataforma online. Ejemplo cuestionarios.

La plataforma está basada en un gestor de contenidos básico, de código abierto, que permite la instalación de módulos, y de programación personalizada relativamente sencilla basada en código PHP. De esta forma, usando las características propias del gestor y las amplias utilidades de módulos disponibles y la cortesía de una empresa local en ceder espacio en su servidor, se ha creado un sistema completo para la realización de los test: midiendo tiempos de conexión, aciertos, dificultad, etc. Todo de forma ya online y estandarizada para poder ser usada cuando el volumen de datos y el avance del proyecto lo determinen. Asimismo, una política de datos abiertos homogeneizará los resultados para poder ser reutilizados. Para ello se usan los formatos más comunes en la actualidad como json, xml y csv.

Los cuestionarios que se usan, están basados y convertidos de formatos físicos a la plataforma manualmente. A nivel técnico se han realizado una serie de configuraciones del sistema para optimizar el trabajo y realizar un desarrollo seguro:

- Sólo se permite realizar los test a usuarios registrados que se les haya dado autorización.

- Existe un control exhaustivo de participantes, de datos, resultados, etc. 
- Está programado de tal forma que un usuario no puede ver de nuevo el test realizado, ni tampoco otros test.

- Está restringido el acceso, incluso a los google bots, para no mostrar resultados o generar ventajas.

- Hay test de diferentes dificultades, y sólo se permite avanzar cuando se han completado otros con conocimientos previos.

- Las preguntas y opciones son siempre de forma aleatoria. El sistema elige " $x$ " preguntas de cada nivel para que nunca se repitan a un mismo usuario.

- Implica tener una gestión de estadísticas, tiempos, fechas, ya optimizada y preparada para el análisis, todo ya en la nube.

\subsection{APLICACIONES}

Todos los juegos y/o aplicaciones se han desarrollado desde cero para este proyecto. Deben ser estructuras estudiadas para que en pantalla aparezcan elementos que fomenten el uso de una habilidad mecánica, y en otros una mental. No siempre es así: en ocasiones la segunda o tercera tarea pueden estar basadas en una dinámica más compleja que sólo despista al jugador de su entrenamiento. A veces, haciéndola sutilmente visible, y en otros momentos, imperceptible. Podría servir de ejemplo un estudiante que es capaz de ver la televisión mientras hace su tarea, o de tocar un instrumento musical mientras lee.

Cada actividad requiere de unas conexiones específicas a nivel neuronal. Añadir otra actividad amplía ese esfuerzo mental, y junto con la multitarea no relacionada, dependiendo en cada caso de su presentación: permitirá una organización perceptiva, agudizará una coordinación visomotriz, mejorará la coordinación óculo-manual, entrenará la visualización espacial, y generará habilidades para una conceptualización abstracta. Por ejemplo, el usuario en una aplicación tendrá que manejar a dos manos dos objetos diferentes en pantalla, mientras resuelve una sencilla operación matemática, o sigue el rastro de unos números, y otra actividad le distrae de todo ello y tendrá que eliminarla.

Las aplicaciones en su mayoría no disponen de sonido, puesto que se pensó podría ser molesto en un entrenamiento continuo. Está pensado que en una misma sala de informática con varios equipos estén varias personas trabajando simultáneamente. Para casos excepcionales donde se trabaja con sonido, o notas musicales, se recomienda el uso de cascos; porque con este tipo de sonidos se juega con las frecuencias de una nota musical, una reverb, o una colocación espacial como en un sistema de grabación binaural. Incluso una persona invidente puede situar un objeto mentalmente usando estas técnicas inmersivas.

El entorno gráfico está también diseñado exprofeso para este proyecto. Debe ser sencillo, claro, minimalista, y generar un ambiente de entrenamiento neutro y agradable. Sólo en algunas aplicaciones, donde casi era obligatorio su uso, se trabaja con iconos o gráficos que dejan de ser simbólicos para ser objetivos.

Una peculiaridad técnica importante es que todas las aplicaciones usan la última tecnología en desarrollo multiplataforma en diseño web adaptativo (adaptable) o Responsive Web Design (RWD); ya no sólo a nivel básico de web o móviles, sino que gracias a las últimas versiones de HML5, JavaScript, y recursos externos de API, DOM, entre otros, las aplicaciones corren sin problema y manteniendo velocidades, tamaños y dificultad en cualquier sistema. Se adaptan incluso a pantalla completa en cualquier navegador, en ordenadores o en dispositivos móviles; casi como una aplicación nativa. Todo ello sin tener que hacer una instalación previa de ninguna herramienta externa. Asimismo, todo el código es relativo a nivel de programación, y usando un estándar complejo de ratios se ajusta a cada pantalla por muy específica que sea; incluso funcionaría en un reloj o un frigorífico. Todo el código está programado en base a código libre -open source-, y como tal es liberado.

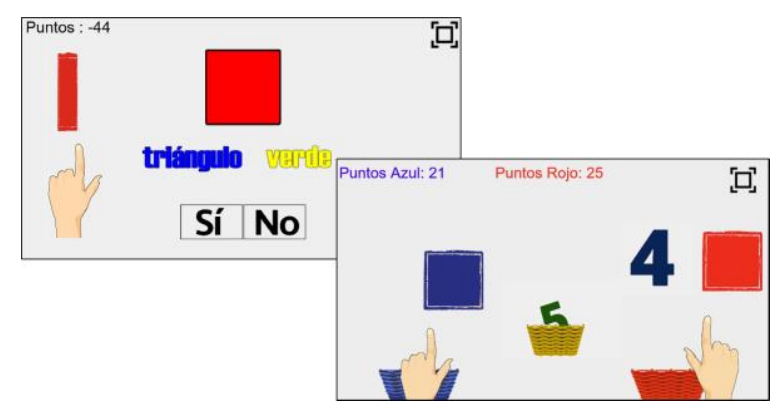

Figura 2. Plataforma online. Ejemplo apps.

En el lado del cliente, la gestión, control de usuarios y datos, estadísticas, test y aplicaciones funcionan en cualquier Sistema Operativo y se visualizan en todos los navegadores web y dispositivos móviles. Cualquier máquina con un intérprete basado en el estándar ECMAScript, e incluso extensiones propias como Opera y el resto de los basados en Chromium, podrá ejecutar estas aplicaciones. A nivel de servidor, en la actualidad, la máquina que centraliza la plataforma está corriendo bajo un sistema LAMP (Linux, Apache, MySQL, PHP), aunque en el futuro puede que se optimicen con servidores específicos para JavaScript, tipo node.js o similares. Por otro lado, la programación se está desarrollando de tal forma que, si fuera necesario, se puede compilar bajo APK, 
IPA, JAR, para la creación de apps nativas más puras y optimizar el rendimiento potencial de un teléfono móvil si ese fuera su destino final.

Hay que llegar a todos los usuarios que tengan interés en participar, sin importarnos su pericia o destreza con los nuevos dispositivos. Se afronta el reto preguntando directamente a los usuarios más neófitos como allanarles el camino, y así se ha creado una línea de trabajo que va desde tutoriales básicos a aplicaciones realmente complejas. La dificultad en muchas de ellas se incrementa basándose en las puntuaciones del jugador, y en otras el grado de dificultad se mantiene más estable, puesto que se trata de un entrenamiento, no simplemente un reto mecánico. Es muy importante que una aplicación fácil, siempre lo sea. Es decir, aunque el usuario vea que existen diferentes elementos nuevos, o pequeños añadidos, la dificultad de esa aplicación concreta ha de ser muy similar. Solo cuando el usuario está preparado, puede elegir una práctica en un nivel superior. Este funcionamiento es importante para no generar frustración en usuarios que comienzan, y para que no exista aburrimiento en un participante avanzado.

Tutoriales: En estas aplicaciones se enseña a un usuario los principios básicos de movimiento que pueda tener o que se vaya a encontrar en los siguientes niveles. Desde hacer un point 'click, manejar un sprite por pantalla, un multitouch básico o incluso usar el teclado del ordenador si la app lo necesita. Se ha probado con usuarios que ni siquiera habían manejado nunca un ordenador o tableta, y los resultados han sido satisfactorios. El equivalente de un clic en una tableta o móvil es compatible con el uso del ratón en un ordenador. El uso de dos dedos, por ejemplo, no es compatible con dos ratones de forma simultánea. Se subsana usando un único ratón en ambos elementos.

Modo Fácil: En este modo se ponen en práctica todos los movimientos aprendidos en los tutoriales, a lo que se añade una pequeña habilidad técnica con algún extra que ya no dependerá de simplemente una pericia con la mecánica del juego, sino que pasa a ser ligeramente mental o intelectual.

Dificultad Media: La interactividad en este apartado ya es manifiesta. En este momento aparecen las primeras multitareas, aunque en un formato muy elemental. Este nivel está pensado para usuarios que raramente podrán pasar al modo siguiente $\mathrm{y}$, por lo tanto, las apps no deben requerir una habilidad mecánica muy alta, aunque la parte mental puede que se acentúe.

Difíciles: Serán evoluciones en su mayoría de la versión "media", donde se intensifica encarecidamente la habilidad técnica, y ligeramente la parte mental. En este apartado, y dependiendo de puntuaciones, las aplicaciones adquieren una endiablada dificultad, para que puedan ser usadas por incluso jugadores de alto nivel.

Como homenaje a ciertos juegos clásicos se han usado sus dinámicas originales para el apartado de habilidad técnica: Pong (1972), Breakout (1976), Monaco GP (1979), Defender (1981), etc. Las mecánicas están basadas en su mayoría en formato Arcade, Sistemas 2D, Perspectivas Cenitales o Matter Physics cuando se requiere realismo con sistemas gravitacionales o rebotes. Es decir, crear un canvas o lienzo donde la física general tenga su lógica y los elementos se comporten lo más parecido a un sistema real: aceleraciones, rotaciones, tiros parabólicos, caídas con inclinaciones, etc.

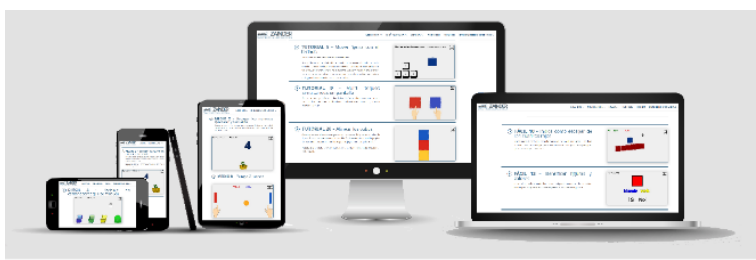

Figura 3. Plataforma online. Visión de los elementos en diferentes plataformas. RWD.

\section{ESTUDIO DE CAMPO}

\subsection{METODOLOGÍA}

El formato será de "Estudio Abierto y Prospectivo", puesto que los participantes conocerán en todo momento la línea de estudio, al igual que los que sólo desarrollen el estudio básico pero no su seguimiento. Lógicamente es un estudio abierto porque el usuario deberá esforzarse en llevar a cabo dicho estudio; y prospectivo porque sólo el tiempo dará resultados válidos a la investigación.

Primero, se somete al voluntario, ya sea de forma online o "in situ" de forma offline, a una serie de test clasificatorios homologados para conocer su punto de partida. Estos test irán acompañados de entrevistas personales.

Segundo, a los asistentes se les suministrarán diferentes aplicaciones específicamente diseñadas para funcionar en cualquier ordenador, tableta o teléfono móvil. Se intentará dejar una o dos tabletas de grandes dimensiones por asociación implicada en el estudio. Se crearán manuales y vídeos explicativos para los coordinadores.

Posteriormente se le invita a participar en el programa con las aplicaciones que se correspondan con su conocimiento, características y habilidades durante 15-45 minutos diarios durante varios meses con: “...herramientas diseñadas específicamente para 
subyugar al cerebro a un -estrés-mediante el uso de actividades multitareas no relacionadas".

Los ensayos tienen una duración de entre 3 y 6 meses. Pasado este tiempo se volverán a realizar test similares a los realizados al comienzo del estudio y se analizarán resultados. Finalmente, se vuelve a tener una entrevista con el usuario y se le examina con un test de nivel similar al de control. Aparte, habrá entrevistas personales, una comunicación fluida y cuestionarios revisados personalizados.

Como parte final del estudio de investigación, se analizarán los resultados y se observará si ha existido algún tipo de mejora global; que no sean simplemente las basadas en el uso de dichas aplicaciones o la práctica de esas habilidades. En paralelo, habrá usuarios de control con similares cuadros clínicos que realicen las evaluaciones periódicamente, pero que no realizarán ninguna otra actividad. Posiblemente se complemente este grupo con usuarios realizando otra tarea para analizar diferentes efectos placebos.

\subsection{MEDIOS}

Tanto para el estado de control, como para el seguimiento de los integrantes los datos están basados en Test Cognitivos Breves (TCB) validados según la Academia Americana de Neurología AAN. Se usan niveles de evidencia de la US Agency for Health Care Policy and Research y la Scottish Intercollegiate Guidelines Network (SIGN). Los grados de evidencia se asignaron a partir de las pautas estándar según el Centre for Evidence-Based Medicine (CEBM) de Oxford [11].

Algunos de los test a usar para el control y seguimiento serán:

- Minimental State Examination (MMSE).

- Test del Reloj.

- SAGE - Self Administered Gerocognitive Examination.

- Figura de Rey Osterrieth.

- EUROTEST y varios diseñados al efecto.

Los test indicados anteriormente, además de crear los puntos de partida, servirán para el control de estado de los participantes a lo largo del tiempo. Se espera que en el avance de las investigaciones se dispongan de más medios, como Electroencefalograma (EEG) en tiempo real, resonancias magnéticas, y el uso de otros equipos.

Hasta el momento hay interesadas diversas asociaciones y colectivos.

- AFA: Asociación de Familiares de Enfermos de Alzheimer y otras demencias de La Rioja.
- ARDEM: Asociación Riojana de Esclerosis Múltiple

- CRUZ ROJA La Rioja.

- OTRAS Asociaciones y Residencias Publicas.

Entre ellos, se crearán y distribuirán los grupos de estudio necesarios, dependiendo de su grado o evolución cognitiva.

Al tratarse de un proyecto de investigación con interacción con seres humanos, éste adquiere unas características especiales. Por ello: se protegerán los derechos y el bienestar de los participantes, asegurando que los riesgos, si existieran, sean razonables comparados con los beneficios potenciales. Asimismo, se garantiza un cumplimiento escrupuloso con el tratamiento de datos personales según RGPD de 2018; y que respetará las normas éticas y legales aplicables de la Declaración de Helsinki [10], donde se recogen los principios básicos para las investigaciones médicas en seres humanos.

\section{CONCLUSIONES}

Se han programado unas herramientas de hardware y software que usan mecánicas sencillas de videojuegos clásicos de ordenador a las que se les han añadido tareas extras, independientes y no relacionadas, con el fin de entrenar al cerebro, sometiéndolo a un trabajo adicional. Se trata de una sobre estimulación o sobre entrenamiento.

Este trabajo presenta dichas herramientas, así como la implantación básica de una completa plataforma que puede ser ampliada a nivel de usuario para completar el sistema de registro y control. También todas las configuraciones, funciones y aplicaciones necesarias de las que van derivando sistemas más complejos multitarea-multitasking, en los que se basará el estudio de campo.

A fecha de junio 2019 ya están funcionales y testeadas el $80 \%$ de las aplicaciones. Existen Betatesters voluntarios chequeando "bajo las condiciones más duras" todas y cada una de las aplicaciones. Se podría decir que fase Alfa está terminada y la fase Beta es funcional. Aun así, quedan varias apps de las que tienen más complejidad bajo una revisión previa. Las aplicaciones con menores opciones están todas online categorizadas como RTM o candidatas definitivas. Paralelamente grupos de usuarios de otras singularidades en cuanto a edad, nivel de estudios, y diferentes impedimentos o discapacidades prueban cada novedad para ajustar la dificultad de partida.

Existe una realimentación de comentarios importante por parte de los implicados para ir implementando nuevas extensiones o eliminar apartados que sean 
excesivamente complejos. Sirva también este feedback para la creación de manuales y hacer hincapié en puntos que para nosotros pasaran más desapercibidos en una primera fase.

La utilización de todas estas herramientas para procesar, administrar, y compartir toda esta información no hubieran sido posibles hace una década. El volumen de datos, sus iteraciones $\mathrm{y}$ relaciones que se generarán será imporatante, y este ya es momento para poder afrontarlo con una viabilidad plena. Se dispone también en este momento de una programación multiplataforma que hace menos de un año aun no tenía las compatibilidades que tienen los nuevos dispostivos. Es el primer momento que nos brinda la tecnología para realizar este tipo de proyectos y estudios de los que vendrán muchos más; y es el momento de utilizarlos.

Como se ha indicado en el punto anterior, tanto fases legales como técnicas están en un punto de desarrollo avanzado. Se podría aventurar que "resueltas", a falta de implementar y completar la plataforma y optimizar la gestión de datos en la nube. Hay usuarios ya trabajando en modo demo, y en las próximas semanas se optimizará el mejor formato para una estratificación correcta; incluir formatos que no puedan sesgar o maquillar los resultados. Paralelamente, se están completando los retoques finales para el soporte de usuarios: generando videos, manuales, etc.

\section{Agradecimientos}

A los desarrolladores de código abierto. Este trabajo se basa en software creado "por y para la comunidad". A los participantes presentes y futuros de este estudio. A la Universidad de La Rioja por la Ayuda a Grupos de Investigación REGI 2018/42.

\section{English summary}

\section{DEVELOPING MULTITASKING APPLICATIONS TO IMPROVE COGNITIVE PERFORMANCE OF PATIENTS WITH DEGENERATIVE DISEASES.}

\author{
Abstract \\ Life expectancy has doubled over four generations, so \\ how to get in good physical and mental faculties is one \\ of the concerns of science. The study of cognitive \\ performance and how to alleviate it, groups diverse \\ areas of work, such us: the brain training projects, \\ through computer applications. Although these
}

studies are still in an embryonic state, since long-term data and results are required, it is clear that this type of activity can contribute positively. This paper presents, how applications for this purpose have been developed that, although based on classical dynamics, are being designed, programmed, adapted and customized for this study. To this end, a set of "non action" games have been programmed with unrelated independent tasks, where the user will train different facets and abilities, submitting the brain to an extra job that is expected to be positive and complements an improvement in the cognitive deterioration process. Simultaneously for the control, monitoring and examination of these competences, an interactive platform has been created on the Internet where samples will be taken via online test of the progress.

Keywords: Multitasking, Degenerative Disease, Brain Training, Superaging.

\section{Referencias}

[1] Manzano S., (2018). Nota de prensa de la Sociedad Española de Neurología SEN. www.sen.es Consulta: 15/06/2019.

[2] López-Bastida J., Serrano-Aguilar P., PeresteloPerez L., Oliva-Moreno J., (2006). "Socialeconomic costs and quality of life of Alzheimer disease in the Canary Islands". Neurology Journal, 67(12), pp. 2186-91.

[3] Villarejo Galende A., Eimil Ortiz M., Llamas Velasco S., Llanero Luque M., López de Silanes de Miguel C., Prieto Jurczynskad C., (2017). "Fundación del Cerebro. Impacto social de la Enfermedad de Alzheimer y otras demencias". Sociedad española de neurología, 10(005), pp. 30-37.

[4] Rogalski E. J., Gefen T., Shi J., Samimi M., Bigio E., Weintraub S., Geula C, Mesulam M., (2013). "Youthful Memory Capacity in Old Brains: Anatomic and Genetic Clues from the Northwestern SuperAging Project". Journal of Cognitive Neuroscience, 25(1), pp. 29-36.

[5] Sun F.W., Stepanovic M.R., Andreano J., Barrett L.F., Touroutoglou A., Dickerson B.C., (2016). "Youthful brains in older adults: Preserved neuroanatomy in the default mode and salience networks contributes to youthful memory in superaging". Journal of Neuroscience, 36(37), pp. $9659-9668$.

[6] Barrios L.J., Minguillón J., Perales F.J., RonAngevin R., Solé-Casals J., Mañanas M.A., 
(2017). "Estado del Arte en Neurotecnologías para la Asistencia y la Rehabilitación en España. Tecnologías Auxiliares, Trasferencia Tecnológica y Aplicación Clínica". RIAI, Revista Iberoamericana de Automática e Informática Industrial, 14(4), pp. 355-361.

[7] Mayas J., Parmentier F.B.R., Andrés P., Ballesteros S., (2014). "Plasticity of Attentional Functions in Older Adults after Non-Action Video Game Training: A Randomized Controlled Trial”, PLoS One, 9(3), pe92269.

[8] Ballesteros S., Prieto A., Mayas J., Toril P., Pita C., Ponce de León L., Reales J.M., Waterworth J.A., (2015). "A randomized controlled trial of brain training with non-action video games in older adults: results of the 3-month follow-up". Frontiers in aging Neuroscience, 7(45), pp. 1-12.

[9] Anguera J.A., Boccanfuso J., Rintoul J.L., AlHashimi O., Faraji F., Janowich J., Kong E., Larraburo Y., Rolle C., Johnston E., Gazzaley A., (2013). "Video Game training enhances cognitive control in older adults". Nature, 501(7465), pp. 97-101.

[10] La Declaración de Helsinki o DoH, (1964). Asociación Médica Mundial (AMM). www.wma.net Versión consultada: última revisión de 2013.

[11] Howick J., Chalmers I., Glasziou P., Greenhalgh T., Heneghan C., Liberati A., Moschetti I., Phillips B., Thornton H., (2011). "The Oxford 2011 Levels of Evidence". Oxford Centre for Evidence-Based Medicine: www.cebm.net Consulta: 15/06/2019.

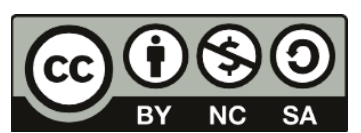

(C) 2019 by the authors. Submitted for possible open access publication under the terms and conditions of the Creative Commons Attribution CC BY-NC-SA 4.0 license (https://creativecommons.org/licenses/bync-sa/4.0/deed.es 